\title{
Evaluation of an evidence-based contraceptive advice line used to support contraceptive provision in primary care
}

\author{
Okunyomibo Graham, Soe Nyunt Aung, Kate Guthrie
}

\begin{abstract}
Background An e-contraceptive advice line (contraceptionadvice.GP@hullpct.nhs) was set up by our unit to support contraceptive provision in primary care. This advice line was for general practitioners (GPs) initially and was then extended to certain pharmacists. All queries were to be answered within 24 hours. A similar e-advice line on emergency contraception for patients seeking advice had been successful in the USA and South Africa.
\end{abstract}

Methods Our aim was to evaluate the contraceptive advice line using standards developed at its inception. A retrospective audit of the queries received by the contraceptive advice line between January and September 2009 was conducted.
Results Sixty-seven queries were received from 30 GPs, nine from practice nurses and none from pharmacists. Some $61 \%$ of the queries were answered within 24 hours and $85 \%$ were answered within 72 hours; $90 \%$ were within the advice line guidelines.

Conclusions The e-mail advice line is a useful, low-cost, well-received support service for GPs. This model could be adopted by other areas wishing to increase contraceptive access in primary care.

Keywords advice line, contraception, general practitioners, pharmacists, primary care

J Fam Plann Reprod Health Care 2010; 36(4): 225-227

(Accepted 8 April 2010)

\section{Introduction}

The concept of an evidence-based contraceptive e-mail advice line (contraceptionadvice.GP@hullpct.nhs) came from the desire to increase access to contraceptive services by supporting general practitioners (GPs) in delivering contraceptive care within their surgeries. It also supported the achievement of Medical Foundation for AIDS and Sexual Health (MedFASH) aspirational standards for 48hour access to contraceptive provision. ${ }^{1}$

The e-mail advice line was designed to be answered in a timely manner for routine queries, thereby reducing frustration from delays with letters and difficulty contacting consultants by phone. In emergencies, immediate telephone support with a senior family planning doctor remained available via secretaries. Our model was based on an existing e-advice line for emergency contraception, although that service is for clients and is little used by service providers. 2,3

\section{Project development}

Technical support from the primary care trust (PCT) information technology (IT) department was sought to determine the feasibility of having an additional shared e-mail inbox for clinicians who will answer the advice line. This was established on personal computers at work. Access via existing work BlackBerry ${ }^{\mathrm{TM}}$ smartphones was not possible however.

The guidelines and protocol for the advice line were set and approved by departmental and PCT clinical governance. A condition for approval was that no patientspecific details were to be given by the clinician requesting advice (for data protection purposes) and that it be made clear this was an advice line, and not a diagnostic or therapeutic service (Figure 1).

Sexual and Reproductive Healthcare Partnership, Conifer House, Hull, UK

Okunyomibo Graham, FRCOG, MFSRH, Subspecialty Registrar

Soe Nyunt Aung, MRCOG, Dip GUM, Specialty Doctor

Kate Guthrie, FRCOG, FFSRH, Clinical Director

Correspondence to: Dr Okunyomibo Graham, Sexual and Reproductive Healthcare Partnership, Conifer House, 32-36 Prospect Street, Hull HU2 8PX, UK. E-mail: yombog@aol.com

\section{Key message points}

- Supporting primary care colleagues using current technology to enhance their contraceptive services is feasible.

- The e-mail advice line is a useful, low-cost, well-received support service suitable for general practitioners, and is a model that could be adopted by other areas wishing to increase contraceptive access in primary care.

\section{Resources}

As the e-mail advice line inbox was shared, the questions were seen by all three clinicians involved and were answered by the first clinician to access the inbox. However, the sent box could not be shared, and so the given advice was copied to the other two clinicians in case secondary questions arose.

GP ensures the query is not for emergency advice (which should continue to be made using immediate telephone support with the senior family planning doctor) or is patient specific

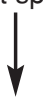

GP e-mails (non-urgent) query to

contraceptionadvice.gp@hullpct.nhs.uk Query must not contain the details of any individual

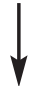

E-mail picked up by one of the three clinicians who have access to the e-mail account within 24 hours

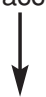

GP receives e-mail response to request for advice within 24 hours

Referral and reply filed for audit purposes

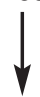

Audit of service to determine timeliness and issues, which can be addressed through training If exclusion breached, GP to be informed immediately

Figure 1 Process for contraception e-mail advice line. GP, general practitioner 


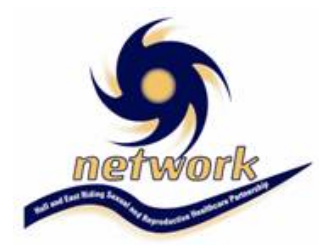

To: GPs/Trainees

Dear Colleague

Contraception Email Advice Line

Not sure what method of contraception is unsafe for someone with cardiomyopathy/stroke/VTE? Or how to manage problematic bleeding patterns on Implanon? Email a tame contraceptive specialist and save your patients a referral with the help of our contraception email advice line.

To enable timely advice on contraceptive issues and reduce the number of referrals to secondary care, we have instituted an advice line on contraceptionadvice.gp@hullpct.nhs.uk with immediate effect. Emails to this address will be answered by email within 24 hours by one of three senior medics within the Sexual and Reproductive Healthcare Partnership based at Conifer House.

Please do not identify individuals in the emails for data protection. This is an advice line, not a diagnostic or therapeutic service.

Please continue to phone if you have urgent queries (via community secretaries on Hull 01482 $336337 / 336378)$.

Please continue to refer by letter if you wish a patient to be seen.

The service will be audited to check it is fit for purpose so please use this email address if you have any immediate comments/feedback on the service.

Yours sincerely

Figure 2 Sample letter announcing the e-mail contraceptive advice line
The aim was to provide evidence-based answers with relevant e-links included when possible.

\section{Implementation}

The guidelines and protocol were reviewed by local GPs who helped design the launch letter (Figure 2) and distribution plan. The development of this e-mail advice line was advertised to National Health Service (NHS) Hull and NHS East Yorkshire GPs by e-mail and through letters to practice managers as a secondary method and for locums. Trainee GPs were informed through the local GP training programme director. The advertisement was sent out at the beginning of January 2009 and the first query was submitted on 23 January 2009.

The e-mail helpline guidelines were as follows:

1 This service is not appropriate for emergency situations. Where a GP has an urgent query they should continue to be addressed via consultant mobile (available from the secretaries on Hull 01482 336377/336378)

2 All requests must be anonymous; patients must not be identifiable.

3 This is not a diagnostic or therapeutic service but an advice-only service.

4 Queries should be replied to within 24 hours.

5 The reply could be from one of three senior clinicians.

6 If exclusion is breached, the GP is to be informed immediately.

The service was audited over the period January 2009 to September 2009 against the standards detailed above. There was no benchmark.

The first request for information from a practice nurse was received on 20 February 2009. The decision was made to answer nurse requests rather than inconvenience patients.
In July 2009 the advice line was offered to NHS Hull pharmacists offering emergency contraception, chlamydia treatment and repeat issuing of contraception by patient group direction.

\section{Results}

During the audit period 67 queries were received, all of which were non-urgent from GP practices. Forty-one $(61 \%)$ were answered within 24 hours and a further 16 $(23 \%)$ were answered within 3 days.

Three clinicians answered the queries; Clinician 1 answered the most queries (38 queries; 57\%); Clinicians 2 and 3 answered four $(6 \%)$ and $14(21 \%)$ queries, respectively. Ten $(15 \%)$ queries were jointly answered by Clinicians 1 and 3 , with Clinicians 2 and 3 jointly answering one query

The majority of the queries $(60 ; 90 \%)$ were within the advice line guidelines; only five were outside the topics covered by the guidelines. These queries were about menopause on two occasions, and GUM, gynaecology and equipment on one occasion each. Two other queries were about contraception but the guideline was breached by the request coming from an administrator and a nurse practitioner. In four cases the e-mails contained patient details.

During the audit period the advice line was accessed by 30 GPs with 53 (79\%) queries, nine nurses with $13(19 \%)$ queries and one administrative staff member on behalf of the GP. The highest number of queries from a single GP was eight, and 18 GPs had one query each. Of the 13 queries received from nurses, three queries were from one nurse, two were from two nurses and remaining six queries were from six different nurses.

\section{Discussion}

Information via e-mail (ceu.members@ggc.scot.nhs.uk) is available from the Clinical Effectiveness Unit (CEU) of the 
Faculty of Sexual and Reproductive Healthcare (FSRH), with a response promised within 5-10 working days. The CEU has a researcher available to co-ordinate answers to queries. Our e-mail advice line was designed for use by local GPs, this later being extended to pharmacists in July 2009 when repeat issuing of hormonal contraception by pharmacists was launched. It is likely that pharmacists did not use the service in the audit period because of this later invitation. A pragmatic approach was adopted for queries received from nurses who accessed the advice line, and their queries were responded to.

Although the advice line guidelines quite clearly stated that only generic discussions (i.e. without patient details) would take place, there were four instances where patient details were included in the queries. These cases were identified and dealt with as per protocol.

This baseline audit showed that we met the standard for replying within 24 hours in only $61 \%$ of cases. However, the protocol design did not take into account the fact that two of the three clinicians had access to the inbox only during office hours, which meant that queries received on a weekend could not be answered by these two clinicians until the following Monday. The majority (84\%) of queries were answered within 3 days. The system also failed when annual/study leave clashed with sick leave. A solution to this problem would be to allow mobile internet access and to have a fixed rota. Without mobile internet access it may be more realistic to state that queries will be answered within two working days and set rotas for answering the queries. If a clinician is unavailable then robust arrangements should be made with a colleague to cover.

Findings from this audit demonstrate that it is possible to support primary care colleagues using current technology to enhance their contraceptive services. Although the numbers in this audit are small, there is enthusiasm to extend the advice line concept to other related services within the Sexual Health Partnership (e.g. community gynaecology, menopause and erectile dysfunction). There have also been enquiries about patients accessing this service.

Frequently asked questions can be used as prompts for topics for in-house training, for primary care updates and for inclusion on the local website.

The queries received were varied, as illustrated by the following examples:

- Contraception following HELLP syndrome

- Contraception in an under-18-year-old with familial hyperlipidaemia

- Intrauterine device timing in the postnatal period

- Intrauterine system and loss of libido

- How to postpone a period in a woman using Logynon ${ }^{\circledR}$

- Duration of combined oral contraceptive use to deal with bleeding on Implanon ${ }^{\circledR}$.

Where possible, the answers were evidence-based using FSRH CEU ${ }^{4}$ guidance or UK Medical Eligibility for Contraceptive Use (UKMEC). ${ }^{5}$ Where the query had previously been dealt with by the CEU this was indicated on the reply and a PDF attachment included. Alternatively relevant sections of text were cut and pasted into the response sent to the GPs so that they could look up the detailed information if they wished and for future reference.

Currently the service is provided with technical support from the PCT and the clinicians 'in the building' between their other routine tasks. If expansion is deemed necessary, or demand for the service increases, specific time will need to be allocated and this will have cost implications.

The e-mail advice line has been well received by users and there has been a lot of positive feedback although no formal audit. Sample comments from users of the e-mail advice line include the following:

"Many thanks. If only all specialties had such a useful quick, practical system for GP enquiries, we may save the NHS a fortune in inappropriate referrals. If you need a feedback audit for your online advice please forward to me as this is the sort of service we find really useful."

"Many thanks for helpful and speedy response. This is an excellent service and well respected by other GPs also."

\section{Recommendations}

The findings from this audit led us to make the following recommendations:

1 Answer queries within two working days.

2 Arrange rotas for answering queries, and if a clinician is unavailable then they should arrange cover.

3 Make a case for funding of mobile internet access.

4 Consider extending the advice line formally to nurse practitioners and to other areas of the partnership (e.g. erectile dysfunction, menopause and community gynaecology).

5 Repeat the audit 6 months after any changes have been implemented.

\section{Conclusions}

The e-mail advice line is a low-cost, useful, support service for GP, nurse practitioner and (potentially) pharmacist contraceptive provision in our locality. This model could be adopted by other areas wishing to increase contraceptive access in primary care.

\section{Statements on funding and competing interests}

Funding None identified.

Competing interests None identified.

\section{References}

1 Medical Foundation for AIDS and Sexual Health. Recommended Standards for Sexual Health Services. 2005. http://www.medfash.org.uk/publications/documents/Recomme nded standards for_sexual health_services.pdf [Accessed 9 December 2009].

2 Wynn L, Trussell J. The morning after on the internet: usage of and questions to the emergency contraception website. Contraception 2005; 72: 5-13.

3 Office of Population Research \& Association of Reproductive Health Professionals. The Emergency Contraception Website. http://ec.princeton.edu/my-ec-story.html [Accessed 9 December 2009].

4 Clinical Effectiveness Unit (CEU) of the Faculty of Sexual and Reproductive Healthcare (FSRH). Various CEU guidance documents available on the FSRH website at http://www.fsrh.org (see Publications/Clinical Guidance).

5 Faculty of Sexual and Reproductive Healthcare. UK Medical Eligibility for Contraceptive Use (UKMEC 2009). 2009. http://www.fsrh.org/admin/uploads/UKMEC2009.pdf [Accessed 9 December 2009].

\section{LETTERS TO THE EDITOR}

Letters to the Editor are welcome and generally should not exceed 600 words or cite more than five references. For comments on material published in the most recent issue of the Journal, correspondence should be received within 4 weeks of dispatch of that Journal to be in time for inclusion in the next issue. When submitting letters correspondents should include their job title, a maximum of two qualifications and their address(es). A statement on competing interests should also be submitted for all letters. Letters may be submitted to the Editor or the Journal Editorial Office (details on page 185). 